\title{
Treatment of masseteric hypertrophy with botulinum toxin a: a retrospective analysis of cases
}

\author{
Diego Barreto Suarez,' Carlos Ruiz Valero, ${ }^{2}$ Diana Dix Luna, ${ }^{3}$ Vanesa Alvarado, ${ }^{3}$ Victoria Mayorga, ${ }^{3}$ \\ Andrea Rivera, ${ }^{3}$ Paula Ramírez, ${ }^{3}$ Álvaro Rodríguez Sáenz ${ }^{4}$ \\ 'DDS, Oral and Maxillofacial Surgery Department, Hospital Simón Bolívar, Colombia \\ ${ }^{2}$ DDS, Oral and Maxillofacial Surgery Department, Hospital San Rafael, Colombia \\ ${ }^{3}$ DDS, Universidad el Bosque, Bogotá, Colombia \\ ${ }^{4}$ DMD, Oral and Maxillofacial Surgery Residency Program, Universidad el Bosque, Bogotá, Colombia
}

Correspondence: Diego Mauricio Barreto Suarez, Oral and Maxillofacial Surgery Department, Universidad El Bosque, Carrera 7 B Bis No I32-II, Bogotá, postal address II0I2I, Colombia, Email aarodriguezs@unbosque.edu.co

Received: August 09, 2018 | Published: January 25, 2019

Copyright@ 2019 Suarez et al. This is an open access article distributed under the terms of the Creative Commons Attribution License, which permits unrestricted use, distribution, and reproduction in any medium, provided the original author and source are credited.

\begin{abstract}
Objective: The purpose of this investigation was to determine the clinical efficiency of the intramuscular injection of botulinum toxin type A as an alternative approach for patients diagnosed with masseteric hypertrophy at Hospital Simón Bolívar and Hospital Universitario Clínica San Rafael in Bogota, Colombia.

Patients and methods: A retrospective case-series investigation was performed. It included healthy patients diagnosed with masseteric hypertrophy and treated by means of an intramuscular injection of botulinum toxin type A, between January 2011 and October 2013. Study variables included patient's medical and dental history, demographics, medication dose and complications. Three outcome variables were measured: Clinical absence or reduction of masseteric hypertrophy, pain and bruxism. Descriptive statistics were computed for each study variable. Results: A total of 20 patients were studied (5 men and 15 women) and the sample's mean age was 29.5 years. After a 6-month follow-up appointment, $15 \%$ of patients presented masseteric hypertrophy, $15 \%$ moderate pain and $85 \%$ mild pain. Only $10 \%$ presented bruxism.
\end{abstract}

Conclusions: Within the limits of this research, botulinum toxin type A is an effective method for the medical treatment of masseteric hypertrophy.

Keywords: Botulinum toxin A, masseteric hypertrophy

\section{Introduction}

In 1880 Legg $^{1}$ published in the Transactions of the Pathological Society of London an article in which he reported the case of a 10-yearold girl with concurrent idiopathic masseter and temporalis muscle hypertrophy. Since then, this rare condition has been sufficiently studied $^{2-4}$ and its treatment ranges from conservative methods such as occlusal splints and muscle relaxants ${ }^{5-7}$ to surgical approaches involving partial resection of the muscle and ostectomy in the area of the muscular insertion. ${ }^{8-10}$

The most recent conservative therapy implemented for the management of masseteric hypertrophy ( $\mathrm{MH})$ is the intramuscular injection of small doses of botulinum toxin type A (BTA), an approach based on the observation that this neurotoxin significantly reduces the size of any muscle by nervous muscle paralysis. ${ }^{11}$ Although Kerner ${ }^{12}$ recognized the possible therapeutic uses of botulinum toxin as early as 1817 , it was not until the york of $\operatorname{Scott}^{13-14}$ throughout the 1970 s and 1980 s that this neurotoxin round its place in clinical medicine.

During the mid and late 1990s BTA was described not only for cosmetic purposes, but also for the treatment of many functional problems. It was, in fact, Moore and Wood ${ }^{15}$ and Smyth ${ }^{16}$ who in 1994 pioneered the use of BTA for the management of masseteric hypertrophy. Their results showed it could be an excellent alternative for the medical management of such condition.

This audit was done to evaluate the clinical efficacy of the intramuscular injection of BTA (Botox ${ }^{\circledR}$, Allergan Inc, Irvine, CA) as an alternative approach to treat patients diagnosed with masseteric hypertrophy (MH) at Hospital Simón Bolivar and Hospital Universitario Clínica San Rafael in Bogotá, Colombia.

\section{Patients and methods}

This research was designed in accordance to the 1975 Declaration of Helsinki on medical protocol and ethics and to local institutional protocols. To be included in the study each subject had to be diagnosed with MH. Exclusion criteria included pregnancy, any serious medical condition, and documented history of allergy to BTA. The study population consisted of all patients round in the database of both hospitals who had completed at least 6 months of follow-up.

In order to achieve our study purpose, we designed a retrospective case series study that screened for patients diagnosed with MH at Hospital Simón Bolivar and Hospital Universitario Clínica San Rafael in Bogotá, Colombia and treated by means of intramuscular injection of BTA between January 2011 and October 2013. Before administration
Submit your Article | www. ologypress.com/submit-article OP $\underset{\text { Press }}{\text { Ology } f \text { in } y \text { witio }}$
Citation: Suarez DB, Valero CR, Luna DD, et al. Treatment of masseteric hypertrophy with botulinum toxin a: a retrospective analysis of cases.J Dent Maxillofacial Res. (2019);2(I): I2-I4. DOI: I0.3088I/jdsomr.00022 
of the drug, a signed written consent form was obtained from every patient. Risks and potential side effects were carefully explained.

BTA $\left(\right.$ Botox $^{\circledR}$, Allergan Inc, Irvine, CA) was supplied by a local representative as a freeze-dried powder of $100 \mathrm{U}$ and was reconstituted with $2 \mathrm{ml}$ of sterile saline solution to a concentration of $10 \mathrm{U} / 0.2 \mathrm{ml}$. The reconstituted medication was used immediately. A total of $25 \mathrm{U}$ of BTA per side was injected using a $1 \mathrm{ml}$ syringe with a 26 -gauge, 0.5 inch needle. The toxin was injected at 2 different points of the masseter muscle within the safety zone originally proposed by Kim et al. ${ }^{17}$

\section{Study variables, data collection and analysis}

A) Medical history: Patient health status was determined according to the American Society of Anesthesiology classification system (ASA I to ASA V). Clinical diagnose of MH was made at the time of initial consultation; B) Dental history: It included presence of bruxism, pain on palpation measured with the Numeric Rating Scale (NRS-11), and oral aperture; C) Demographics: Gender and age at the time of the intervention was recorded; D) Dose: 25 units of BTA were injected on each masseter, for a total of 50 units per patient, and E) Complications: Any complication related to the intramuscular administration of BTA was recorded. Three outcome variables were measured: Clinical absence or reduction of masseteric hypertrophy, pain, and bruxism. Data was collected from November to December 2013 and analyzed in January 2014. Descriptive statistics were computed for each study variable using the Microsoft Excel ${ }^{\circledR}$ Software.

\section{Results}

Table 1 reveals that a total of 20 patients (mean age 29.5 years), 15 women and 5 men, met the inclusion criteria for the study. The medical history revealed that all subjects were classified as ASA I and had bruxism or parafunctional habits. On initial consultation and at the operative appointment, severe pain on palpation was documented on all patients. At a 3-month follow-up appointment (Table 2), 15\% of patients still presented $\mathrm{MH}$ and pain was classified as moderate on $100 \%$ of subjects. At a 6-month follow-up appointment, $15 \%$ of patients presented $\mathrm{MH}, 15 \%$ moderate pain and $85 \%$ mild pain. Only $10 \%$ presented bruxism (Table 3 ). No complications were documented.

Table I Characteristics of patients at initial consultation

\begin{tabular}{ll}
\hline Variables & Result \\
\hline Total of Patients & 20 \\
Women & 15 \\
Men & 5 \\
Overall Mean Age & 29.5 \\
Women & 29 \\
Men & 30 \\
Clinical Findings & \\
Bruxism & $20(100 \%)$ \\
Grinding Surfaces & $20(100 \%)$ \\
Pain on Masseter Palpation (NRS-II) & $20(100 \%)$ \\
I-3 (Mild Pain) & $0(0 \%)$ \\
4-6 (Moderate Pain) & $0(0 \%)$ \\
7-I0 (Severe Pain) & $20(100 \%)$ \\
\hline
\end{tabular}

Table 2: Efficacy of BTA in patients with MH seen at Hospital Simón Bolivar and Hospital Universitario Clínica San Rafael in Bogota, Colombia at a 3-month Follow-Up

\begin{tabular}{ll}
\hline Variables & Result \\
\hline $\begin{array}{l}\text { Overall Reduction of Masseteric Hypertrophy } \\
(75 \%)\end{array}$ & 15 \\
$\begin{array}{l}\text { Overall Persistence of Masseteric Hypertrophy } \\
\text { I } 5 \%)\end{array}$ & 5 \\
Overall Reduction of Bruxism & $16(80 \%)$ \\
Overall Persistence of Bruxism & $4(20 \%)$ \\
$\begin{array}{l}\text { Pain on Masseter Palpation (NRS-I I) } \\
\text { (I00\%) }\end{array}$ & 20 \\
I-3 (Mild Pain) & $0(0 \%)$ \\
$4-6$ (Moderate Pain) & 20 \\
I $00 \%)$ & $0(0 \%)$ \\
\hline
\end{tabular}

Table 3: Efficacy of BTA in patients with MH seen at Hospital Simón Bolivar and Hospital Universitario Clínica San Rafael in Bogota, Colombia at a 6-month Follow-Up

\begin{tabular}{ll}
\hline Variables & Result \\
\hline Overall Reduction of Masseteric Hypertrophy (75\%) & 17 \\
Overall Persistence of Masseteric Hypertrophy (I5\%) & 3 \\
Overall Reduction of Bruxism & $18(90 \%)$ \\
Overall Persistence of Bruxism & $2(10 \%)$ \\
Pain on Masseter Palpation (NRS-II) (I00\%) & 20 \\
I-3 (Mild Pain) (85\%\%) & 17 \\
$4-6$ (Moderate Pain) & $3(15 \%)$ \\
7-I0 (Severe Pain) & $0(0 \%)$ \\
\hline
\end{tabular}

\section{Discussion}

The purpose of this audit was to determine the clinical efficacy of the intramuscular injection of BTAas an alternative approach to treat patients diagnosed with MH at Hospital Simón Bolivar and Hospital Universitario Clínica San Rafael in Bogota, Colombia between January 2011 and October 2013 with a 6-month follow-up.

$\mathrm{MH}$ is defined as an asymptomatic enlargement of one or both masseter muscles. Its etiology is not completely understood, although certain conditions such as bruxism, malocclusion, clenching and temporomandibular joint disorders have been linked to it. Most cases are symmetric and bilateral. ${ }^{18}$ In the case series we present, all patients presented with bilateral $\mathrm{MH}$, which was mainly related to bruxism. We studied a sample of 20 patients ( 15 women and 5 men) who debuted with severe masseteric pain, whose condition was unsuccessfully treated by different other conservative methods such as local heat, occlusal splints, and medication. ${ }^{5-7}$

Other studies on the use of BTA to treat $\mathrm{MH}^{15-18}$ have proven this to be a reliable method to treat the condition. In our series, at a 6-month follow-up we found that this neurotoxin, when properly injected, constitutes a valuable clinical, non-invasive tool to treat patients with MH. Injection of BTA was effective in $75 \%$ of the patients as it related 
to reduction of $\mathrm{MH}$. All patients related a significant reduction of pain with no complications at all. In this report, we have documented the successful use of BTA in the treatment of 20 patients with $\mathrm{MH}$ Important esthetic improvement was maintained in all 20 patients.

\section{Conflict of interests}

The authors report no conflicts of interest related to this study

\section{Acknowledgements}

The authors express their gratitude to Dr Luis Fernando Gamboa, Diana Dix, and Jaime Castro-Núñez for their assistance during the preparation of this paper.

\section{References}

I. Legg W. Enlargement of the temporal and masseter muscle in both sides. Trans Pathol Soc Lond. 1880;3 I:36 I-364.

2. Barton R. Benign masseteric hypertrophy: a syndrome of importance in the differential diagnosis of parotid tumors. JAMA. I957; | 64(I5): I646-1647.

3. Kessel L. Benign bilateral masseteric hypertrophy with temporal muscle involvement. Oral Surg 1970;30(4):450-453.

4. Addante RR. Masseter muscle hypertrophy: report of a case and literature review.J Oral Maxillo fac Surg. 1994; 52(I I) I I99-1 202.

5. Black MJ, Schloss MD. Masseteric muscle hypertrophy. J Otolaryngol. 1985; |4(3):203-205.

6. Bertram S, Rudisch A, Bodner G, et al. Effect of stabilization-type splints on the asymmetry of masseter muscle sites during maximal clenching.J Oral Rehabil 2002;29(5):447-45I.
7. Sannomiya EK, Goncalves M. Masseter muscle hypertrophy: case report Braz Dent. J 2006; 17(4):347-350.

8. Gurney CE. Chronic bilateral benign hypertrophy of the masseter muscles. Am J Surg. 1947;73(1): 137-139.

9. Beckers HL. Masseteric muscle hypertrophy and its intraoral surgical correction. J Maxillofac Surg. 1977;5(I):28-35.

10. Chee LF, Fei YJ. Surgical correction of masseter muscle hypertrophy by an intraoral approach. J Oral Maxillofac Surg 1989;47(8):883-885.

II. Wheeler A, Smith HS. Botulinum toxins: mechanisms of action, antinoception and clinical applications. Toxicology 20I3;306:I24-I46.

12. Erbguth FJ, Naumann M. Historical aspects of botulinum toxin: Justinus Kerner (I 786-1862) and the "sausage poison".Neurology. I999;53(8):I850 1853.

13. Scott $A B$, Rosenbaum A, Collins $C C$. Pharmacologic weakening of extraocular muscles. Invest Ophthalmol. 1973; I2(I2):924-927.

14. Scott $A B$. Botulinum toxin injection of eye muscles to correct strabismus. Trans Am Ophthalmol Soc. 1981;79: 734-770.

15. Moore AP,Wood GD.The medical management of masseteric hypertrophy with botulinum toxin type A. Br J Oral Maxillofac Surg. 1994;32(I):26-28.

16. Smyth AG: Botulinum toxin treatment of bilateral masseteric hypertrophy. Br J Oral Maxillofac Surg. 1994; 32(I):29-33.

17. Kim NH, Chung JH, Park RH, et al. The use of botulinum toxin type $\mathrm{A}$ in aesthetic mandibular contouring. Plast Reconstr Surg. 2005; I I 5(3): 919-930.

I8. Mandel L, Tharakan M: Treatment of unilateral masseteric hypertrophy with botulinum toxin: case report. J Oral Maxillofac Surg. 1999;57(8): 10171019. 